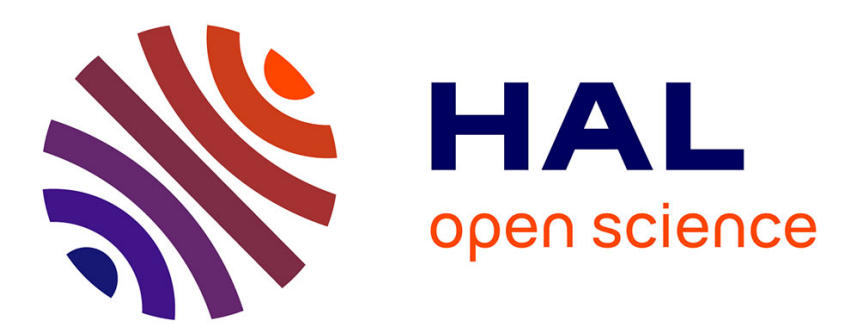

\title{
Transmission-Based Imaging of an Electrically Small Aperture in a Shielded System
}

\author{
Andrea Cozza, Florian Monsef
}

\section{To cite this version:}

Andrea Cozza, Florian Monsef. Transmission-Based Imaging of an Electrically Small Aperture in a Shielded System. IEEE Antennas and Wireless Propagation Letters, 2015, 14, pp.591-594. 10.1109/LAWP.2014.2374699 . hal-01085996

\section{HAL Id: hal-01085996 https://hal.science/hal-01085996}

Submitted on 21 Nov 2014

HAL is a multi-disciplinary open access archive for the deposit and dissemination of scientific research documents, whether they are published or not. The documents may come from teaching and research institutions in France or abroad, or from public or private research centers.
L'archive ouverte pluridisciplinaire HAL, est destinée au dépôt et à la diffusion de documents scientifiques de niveau recherche, publiés ou non, émanant des établissements d'enseignement et de recherche français ou étrangers, des laboratoires publics ou privés. 


\title{
Transmission-Based Imaging of an Electrically Small Aperture in a Shielded System
}

\author{
Andrea Cozza, Senior Member, IEEE, Florian Monsef, Member, IEEE
}

\begin{abstract}
The possibility of imaging the position and nature of apertures in a metallic shielding is discussed. Synthetic images are obtained from signals induced at the interior of the shielding by a set of plane waves impinging on it along different directions of arrival. The proposed technique works analogously to inverse synthetic aperture radar imaging, but is based on signals related to transmitted rather than scattered waves. A theoretical analysis of the features of the images associated to electric and magnetic coupling is presented.
\end{abstract}

Index Terms-Shielding, coupling, small apertures, imaging techniques, radiated tests.

\section{INTRODUCTION}

Sensitive electronic systems are routinely protected by means of metallic shields, intended to reduce the strength of voltages and currents induced along electric/electronic circuits [1]. While shields must necessarily present some apertures, e.g., cooling vents [2] and holes for passing cables, it may happen that unwanted apertures appear as a result of a faulty manufacturing process (e.g., unperfect soldering of two plates) or as the shield deteriorates over time (e.g., because of rust). Since their presence has a direct impact on the effectiveness of the shielding, it is of practical importance to have a procedure for the identification and localization of such apertures.

Visual inspection as well as scanning the surface of the shield with a localized [3] or distributed [4] source of electromagnetic radiation are two pragmatic, yet cumbersome, solutions that are unfortunately not automatic neither in their operation, nor in the quality of their analysis.

Submitting a system under test (SUT) to electromagnetic waves is another potential solution for identification, as they couple through the apertures and induce measurable alterations in the nominal functioning of the SUT. Locally-plane waves are the main tool employed for this task, since they can be easily generated, but they have a major drawback : they do not provide any spatial resolution good enough to localize the position of the aperture, while only their direction of incidence and polarization are known. Ensuring spatial resolution is a fundamental requirement in order to have an automatic procedure to localize unwanted apertures. A previous attempt was presented in [5], based on near-field scans.

An alternative approach can be found by adapting certain ideas applied in radar imaging. In this context, synthetic

Part of the work here reported was funded by the French National Research Agency through the grant ANR-12-ASTR-0005-01, MIMOCHIC project, within the framework of the 2012 ASTRID program.

A. Cozza and F. Monsef are with the Département de Recherche en Électromagnétisme, Laboratoire des Signaux et Systèmes (L2S), UMR 8506 SUPELEC - Univ Paris-Sud - CNRS, 3 rue Joliot-Curie, 91192 Gif-sur-Yvette, France. Contact e-mail: andrea.cozza@supelec.fr. images of unknown systems are generated by submitting them to a series of plane waves, with the aim to gain access to spatial information, e.g., the shape of a scatterer. Inverse synthetic aperture imaging (ISAR) [6] is indeed a measurement-based procedure that is meant to extract automatically the features of an SUT. Of special importance, is the fact that the quality and accuracy of the results thus obtained can be set a priori, thus allowing a systematic and reproducible procedure.

In the rest of this paper we study the possibility to adapt the ideas underlying ISAR to the problem of identifying apertures in a metallic shield. The dependence existing between the nature of the images, the features of the aperture and the type of electromagnetic coupling is explored theoretically for a cylindrical SUT.

\section{LOCATING COUPLING APERTURE FROM RX SIGNALS}

In order to understand how an aperture can be imaged, we first consider the case of a receiver sensitive to the $\hat{z}$-oriented component of the electric field. The receiver is found in free space at the position $\boldsymbol{r}_{c}$ and is illuminated by a plane wave impinging from a direction $\hat{\boldsymbol{k}}$, such that $\hat{\boldsymbol{k}} \cdot \hat{\boldsymbol{z}}=0$; the plane wave is $\hat{z}$-polarized (TM incidence). The received signal can thus be written as

$$
G(\hat{\boldsymbol{k}})=C \mathrm{e}^{-\mathrm{j} k_{o} \hat{\boldsymbol{k}} \cdot \boldsymbol{r}_{c}},
$$

where $C$ is a constant. Information about the position $\boldsymbol{r}_{c}$ is thus mapped into phase-shift angles. A similar property is shared by Fourier-transform pairs, e.g., applied between variables $x$ and $p$

$$
\mathcal{F}\left\{\delta\left(x-x_{c}\right)\right\}(p)=\mathrm{e}^{-\mathrm{j} p x_{c}},
$$

where $x_{c}$ is a spatial offset and $\delta(\cdot)$ is Dirac's delta distribution. It could therefore be possible to infer the position $\boldsymbol{r}_{c}$ by inverse-transforming the received signals $G(\hat{\boldsymbol{k}})$, as

$$
g(\boldsymbol{s})=\int \mathrm{d} \hat{\boldsymbol{k}} G(\hat{\boldsymbol{k}}) \mathrm{e}^{+\mathrm{j} k_{o} \hat{\boldsymbol{k}} \cdot \boldsymbol{s}},
$$

though this operation does not strictly correspond to an inverse Fourier transform, as it should have required all wavenumbers in order to yield a true Fourier-transform pair, and consequently a perfect localization of the aperture: (3) only involves propagation vectors of modulus equal to $k_{o}$. In fact, for the case of the ideal receiver in (1), (3) yields a spatial distribution in the spatial variable $s$

$$
g(\boldsymbol{s})=C \mathrm{~J}_{0}\left(k_{o}\left\|\boldsymbol{s}-\boldsymbol{r}_{c}\right\|\right),
$$

with $\mathrm{J}_{0}(\cdot)$ Bessel's function of the first kind of zero-th order. This image, also known as point-spread function, is indeed centered over the receiver position $\boldsymbol{r}_{c}$. 


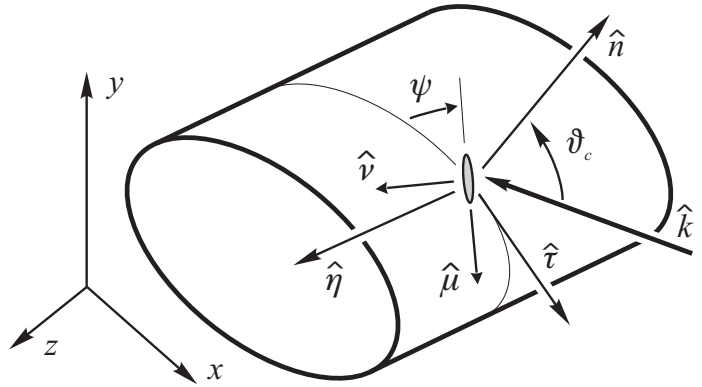

Fig. 1: The problem under consideration : a shielded SUT submitted to plane waves impinging from the direction $\hat{k}$, coupling through an aperture with principal axis $\hat{\boldsymbol{\mu}}$ and $\hat{\boldsymbol{\nu}}$.

These concepts and limitations are fundamentally the same that have been exploited for decades for inverse synthetic aperture radar imaging techniques [6]. Spatial resolution can be improved by using multi-frequency excitations, but this possibility will not be treated in this paper, since our main goal is to study the main features of the images computed from data generated by waves coupling through a small aperture. Our derivation is limited to the case of a cylindrical SUT, which can be treated as a $2 \mathrm{D}$ configuration. A harmonic dependence will be assumed and the corresponding frequency variable will be omitted in subsequent derivations.

\section{COUPling THROUGH A SHIELDING}

In the context of aperture imaging, the role of receiver is played by any electrical port within the internal region protected by the shielding, where an electrical quantity $G(\hat{\boldsymbol{k}})$ is measured, e.g., a voltage across a transmission line over a PCB or a signal received by a probe, induced by a plane wave impinging along the direction $\hat{\boldsymbol{k}}$. A fundamental requirement is that $G(\hat{\boldsymbol{k}})$ be linearly related to the impinging field.

This signal can be related to impinging waves thanks to Bethe's theory for small apertures. Bethe showed in [7] how to model the coupling of an impinging wave onto an electrically small aperture in a shielding. His model allows using a topological approach [1], by separating the problem into two parts: 1) external coupling, based on the definition of equivalent dipole moments representing the coupling of the external wave through the aperture; 2) internal coupling related to the excitation of electromagnetic energy propagating from the equivalent sources to the actual receiver. This approach has been extensively applied to a variety of apertures, with good accuracy with respect to experimental results [1].

Bethe's model defines electric and magnetic dipole moments for the equivalent sources as linearly dependent on the impinging electric and magnetic fields through two dyadic functions, $\boldsymbol{\alpha}^{e}$ and $\boldsymbol{\alpha}^{m}$, usually referred to as the electric and magnetic polarizabilities of the aperture

$$
\begin{aligned}
\boldsymbol{p}_{e} & =\left[\alpha_{n}^{e} \hat{\boldsymbol{n}} \hat{\boldsymbol{n}}\right] \cdot \boldsymbol{E}=\hat{\boldsymbol{n}} \alpha_{n}^{e} E^{\perp} \\
\boldsymbol{p}_{m} & =\left[\alpha_{\mu}^{m} \hat{\boldsymbol{\mu}} \hat{\boldsymbol{\mu}}+\alpha_{\nu}^{m} \hat{\boldsymbol{\nu}} \hat{\boldsymbol{\nu}}\right] \cdot \boldsymbol{H}=\boldsymbol{\alpha}^{m} \boldsymbol{H}^{/ /},
\end{aligned}
$$

where $\hat{\boldsymbol{\mu}}$ and $\hat{\boldsymbol{\nu}}$ are the principal axes of the magnetic polarizability matrix (cf. Fig. 1); $E^{\perp}$ stands for the normal component of the impinging electric field while $\boldsymbol{H}^{/ /}$is the portion of magnetic field tangential to the aperture plane.

In practice, the orientation of the principal axes relative to the transversal plane $x y$ is not known. We therefore consider a general setting where testing plane waves propagate along the $x y$ plane. The natural associated local reference for modelling the coupling at the aperture consists of tangential unit vectors $\hat{\boldsymbol{\tau}}$ and $\hat{\boldsymbol{\eta}}$ and the outward unit vector $\hat{\boldsymbol{n}}$, as defined in Fig. 1 . Since TE and TM scenarios will be defined w.r.t. the $x y$ plane, the actual magnetic polarizability will take the shape of a full $2 \times 2$ matrix

$$
\boldsymbol{\alpha}^{m}=\boldsymbol{R} \boldsymbol{\alpha}_{o}^{m} \boldsymbol{R}^{T},
$$

where $\boldsymbol{\alpha}_{o}^{m}=\operatorname{diag}\left\{\alpha_{\mu}^{m}, \alpha_{\nu}^{m}\right\}$, while $\boldsymbol{R}$ is the rotation matrix for an angle $\psi$ that allows passing from the local transversal vectors $(\hat{\boldsymbol{\tau}}, \hat{\boldsymbol{\eta}})$ tangential to the shielding at the aperture position, to the principal axis $(\hat{\boldsymbol{\mu}}, \hat{\boldsymbol{\nu}})$ of the magnetic polarizability.

For internal transfer functions $Y_{m}^{\tau, \eta}$ and $Y_{e}$ (cf. Fig. 2), relating each scalar component of Bethe sources and their effect on receiving-port signals, $G(\hat{\boldsymbol{k}})$ can be expressed as

$$
G(\hat{\boldsymbol{k}})=\left[Y_{m}^{\tau}, Y_{m}^{\eta}\right] \boldsymbol{\alpha}^{m} \boldsymbol{H}^{/ /}\left(\boldsymbol{r}_{c}\right)+Y_{e} \alpha_{n}^{e} E^{\perp}\left(\boldsymbol{r}_{c}\right) .
$$

Because of the assumption of an electrically small aperture, and thus weakly coupling between the internal and external spaces, the internal transfer functions can be expected to be independent from the direction of arrival of the testing wave, as well as its polarization. As a result, (7) implies that the received signal $G(\hat{\boldsymbol{k}})$ behaves as a proxy of Bethe sources, even though they are not directly accessible.

In a test scenario, the SUT would be submitted to plane waves with two complementary polarization settings, usually referred to as TM and TE incidence. Since all field-related quantities are sampled at the the aperture position, the spatial variable will be omitted, in order to simplify our notations. With reference to Fig. 2, the electric and magnetic fields of the two settings would be

$$
\begin{array}{cc}
\boldsymbol{E}_{T M}=E_{T M} \hat{\boldsymbol{\eta}} & \boldsymbol{H}_{T M}=-\left(E_{T M} / \zeta\right) \hat{\boldsymbol{\vartheta}}_{c} \\
\boldsymbol{E}_{T E}=-E_{T E} \hat{\boldsymbol{\vartheta}}_{c} & \boldsymbol{H}_{T E}=-\left(E_{T E} / \zeta\right) \hat{\boldsymbol{\eta}},
\end{array}
$$

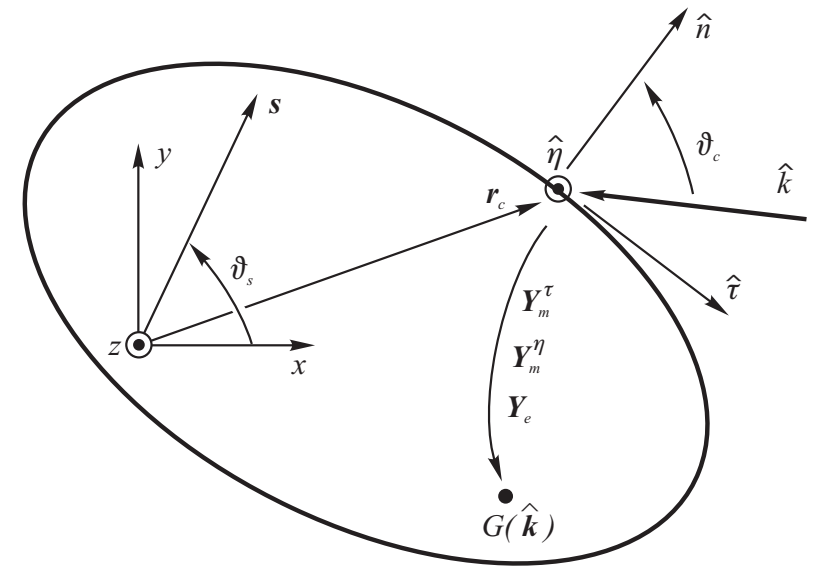

Fig. 2: Internal coupling : Bethe sources for a plane wave impinging along $\hat{\boldsymbol{k}}$ induce an electrical quantity $G(\hat{\boldsymbol{k}})$ measured, e.g., along a circuit within the shielded region. 
i.e., with $\zeta$ the free-space wave impedance and

$$
\boldsymbol{H}^{\|}\left(\boldsymbol{r}_{c}\right)=\left[\begin{array}{c}
H_{T M}^{\tau} \\
H_{T E}^{\eta}
\end{array}\right]=\left[\begin{array}{c}
H_{T M} \cos \vartheta_{c} \\
H_{T E}
\end{array}\right],
$$

and

$$
E^{\perp}=E_{T E} \sin \vartheta_{c} .
$$

Merging these two last expressions with (7), an image of the aperture coupling can be obtained by applying (3) to

$$
G(\hat{\boldsymbol{k}})=\left[Y_{m}^{\tau}, Y_{m}^{\eta}\right] \boldsymbol{\alpha}^{m}\left[\begin{array}{c}
H_{T M} F_{c}(\hat{\boldsymbol{k}}) \\
H_{T E} F_{i}(\hat{\boldsymbol{k}})
\end{array}\right]+Y_{e} \alpha_{n}^{e} E_{T E} F_{s}(\hat{\boldsymbol{k}}) .
$$

Three different kinds of angular functions are required in (11) in order to explain the way an external field couples to internal electric quantities, and ultimately lead to an image of the aperture. Given their different angular dependence, the images obtained by means of (3) will differ. These three patterns are

$$
\begin{aligned}
& F_{i}(\hat{\boldsymbol{k}})=1 \\
& F_{c}(\hat{\boldsymbol{k}})=\cos \left[\vartheta_{c}(\hat{\boldsymbol{k}})\right] \\
& F_{s}(\hat{\boldsymbol{k}})=\sin \left[\vartheta_{c}(\hat{\boldsymbol{k}})\right]
\end{aligned}
$$

defined for $\vartheta_{c} \in[-\pi / 2, \pi / 2]$, i.e., for a plane wave impinging from the half-space in front of the aperture plane. In the following, we will assume that coupling from directions outside this angular span is negligible, i.e., modeling only line-of sight contributions.

\section{IMAGES OF BETHE SOURCES}

Once the angular response of each Bethe source is known, it is possible to compute the images obtained from the application of (3). We will assume in the following that the aperture is found at $\boldsymbol{r}_{c}=0$, and $\hat{\boldsymbol{n}}=\hat{\boldsymbol{y}}$, with no loss of generality. For any of the responses in (12), here referred to as a generic $F\left(\vartheta_{c}\right)$, its corresponding image is

$$
g(\boldsymbol{s})=\int_{-\pi / 2}^{\pi / 2} \mathrm{~d} \vartheta_{c} F\left(\vartheta_{c}\right) \mathrm{e}^{-\mathrm{j} k_{o} \boldsymbol{s} \cdot \hat{\boldsymbol{k}}\left(\vartheta_{c}\right)},
$$

where the integral is limited to the interval $[-\pi / 2, \pi / 2]$, because of the assumed negligible contribution from non lineof-sight directions of incidence.

The integral (13) operates on an angular response similarly to Fourier transform. As a result, since all of the (12) are real-valued functions, their images $g(s)$ can be expected to be Hermitian functions, i.e., $g(-s)=g^{*}(s)$, where ${ }^{*}$ is the complex conjugate. Hence, the imaginary part of each image must display an odd symmetry, whereas for the real part an even symmetry will be observed.

Recalling Jacobi-Anger expansion

$$
\mathrm{e}^{j z \cos \vartheta}=\sum_{n=-\infty}^{\infty} \mathrm{j}^{n} \mathrm{~J}_{m}(z) \mathrm{e}^{\mathrm{j} n \vartheta}
$$

and introducing the complex coefficients

$$
a_{n}=c_{n}+\mathrm{j} s_{n}=\int_{-\pi / 2}^{\pi / 2} \mathrm{~d} \vartheta_{c} F\left(\vartheta_{c}\right) \mathrm{e}^{-\mathrm{j} n \vartheta_{c}}
$$

(13) can be recast as

$$
\begin{aligned}
& g(s)=c_{0} \mathrm{~J}_{0}\left(k_{o} s\right) \\
& +2 \sum_{n=0}^{\infty} w_{n} \mathrm{~J}_{n}\left(k_{o} s\right)\left[c_{n} \cos \left(n \vartheta_{s}\right)+s_{n} \sin \left(n \vartheta_{s}\right)\right]
\end{aligned}
$$

with $\vartheta_{s}$ the polar angle of $s$, as shown in Fig. 2, while

$$
w_{n}=\left\{\begin{array}{cl}
-1 & \text { even } n \\
(-1)^{(n-1) / 2} & \text { odd } n
\end{array}\right.
$$

The $a_{n}$ coefficients for the three patterns (12) are

$$
\begin{aligned}
a_{n}^{i} & =(\pi / 2) \operatorname{sinc}(n \pi / 2) \\
a_{n}^{c} & =-\frac{2 \cos (n \pi / 2)}{n^{2}-1} \\
a_{n}^{s} & =\mathrm{j} n a_{n}^{c}
\end{aligned}
$$

where the labels $i, c$ and $s$ refer to the three Bethe patterns in (12), in this order.

The images of the three coupling mechanisms (12) can be expressed as series expansions, with the isotropic pattern for TE magnetic coupling giving

$$
\begin{aligned}
& \operatorname{Re}\left\{g_{i}(s)\right\}=(\pi / 2) \mathrm{J}_{0}\left(k_{o} s\right) \\
& \operatorname{Im}\left\{g_{i}(s)\right\}=\sum_{\text {odd } n>0}(2 \mathrm{j} / n) \mathrm{J}_{n}\left(k_{o} s\right) \cos \left(n \vartheta_{s}\right),
\end{aligned}
$$

while for TM magnetic coupling

$$
\begin{aligned}
\operatorname{Re}\left\{g_{c}(s)\right\} & =2 \mathrm{~J}_{0}\left(k_{o} s\right)+ \\
& +4 \sum_{\text {even } n>0} \frac{(-1)^{n / 2}}{n^{2}-1} \mathrm{~J}_{n}\left(k_{o} s\right) \cos \left(n \vartheta_{s}\right) \\
\operatorname{Im}\left\{g_{c}(s)\right\} & =\mathrm{j} \pi \mathrm{J}_{1}\left(k_{o} s\right) \cos \left(\vartheta_{s}\right),
\end{aligned}
$$

and for TE electric coupling

$$
\begin{aligned}
& \operatorname{Re}\left\{g_{s}(s)\right\}=4 \sum_{\text {even } n>0} \frac{n(-1)^{n / 2}}{n^{2}-1} \mathrm{~J}_{n}\left(k_{o} s\right) \sin \left(n \vartheta_{s}\right) \\
& \operatorname{Im}\left\{g_{s}(s)\right\}=\mathrm{j} \pi \mathrm{J}_{1}\left(k_{o} s\right) \sin \left(\vartheta_{s}\right) .
\end{aligned}
$$

The three images are shown in Fig. 3 and present a spatial resolution approximatively equal to half a wavelength. In practice, the shape of the different spots at the aperture position $\boldsymbol{r}_{c}=0$ can present different widths along $\hat{\boldsymbol{\tau}}$ and $\hat{\boldsymbol{n}}$, as for the case of the real part of the image for TM magnetic coupling.

For the two magnetic-coupling images, it can be noticed how their imaginary parts are characterized by a pair of peaks with opposite signs, along $\hat{\boldsymbol{n}}$, implying that the orientation of the aperture can be inferred from these images. Furthermore, their real parts feature a peak centered over the position of the aperture. Conversely, the electric coupling has a complementary behavior, with both real and imaginary parts equal to zero at the aperture position, thus allowing separating their respective contributions.

Knowledge of the properties of the images for the three coupling contributions can be exploited in order to interpret the overall results (11) that would be obtained from experimental tests. For instance, by submitting the SUT to a series of plane-wave excitations along all directions of arrival over the azimuthal plane $x y$, with TE incidence and an electric field 


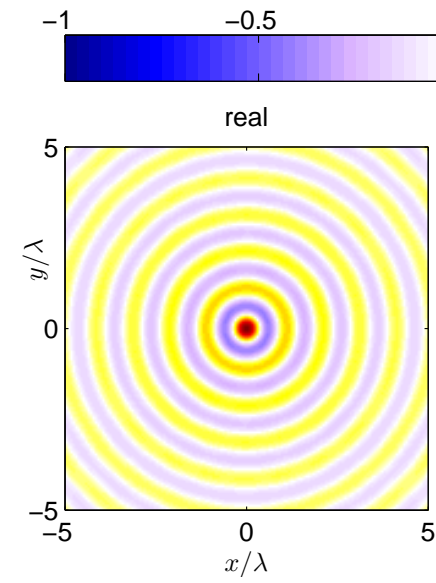

0
1 $\quad 0.5$
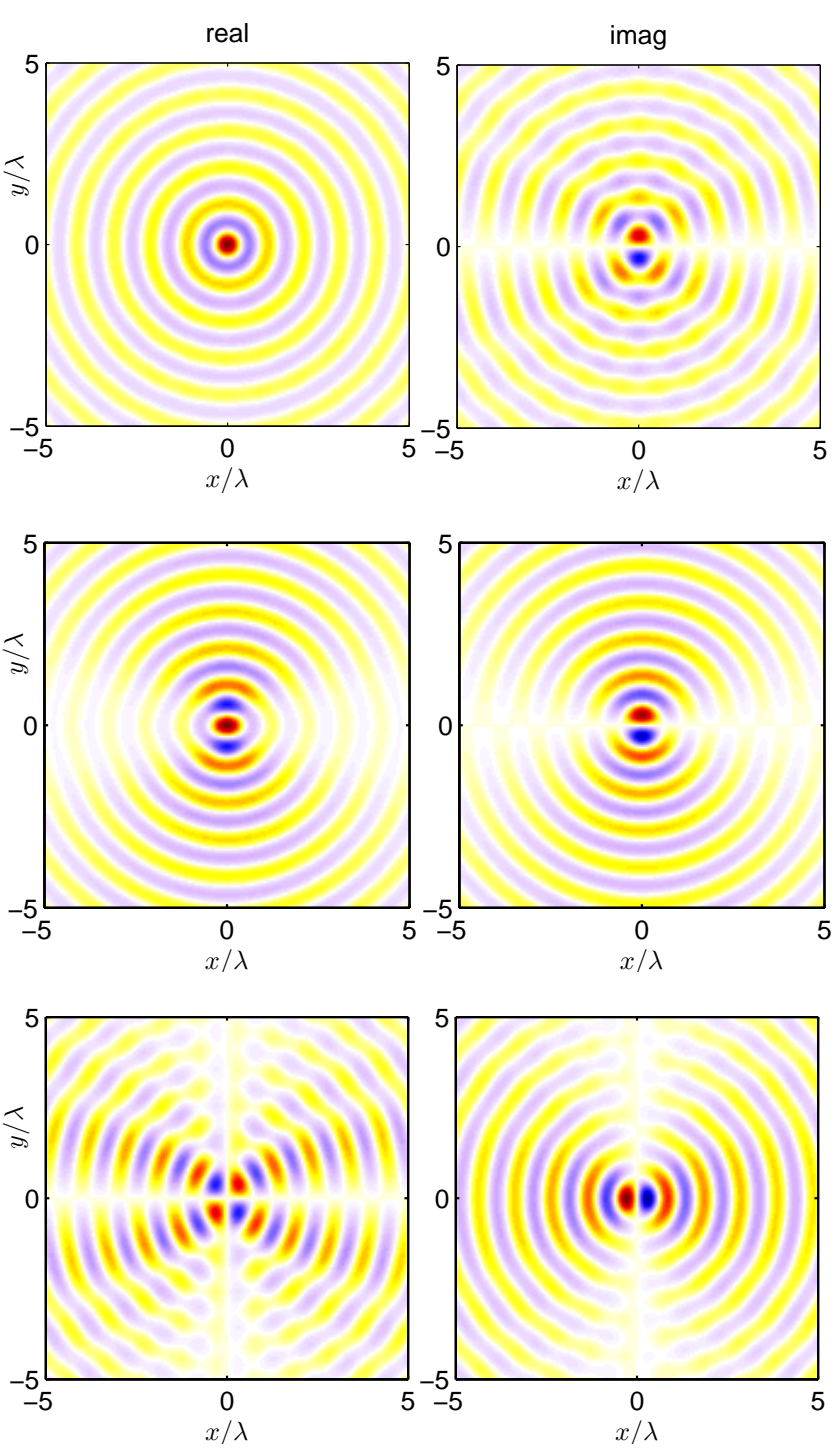

Fig. 3: Real (left-hand side) and imaginary parts (right-hand side) of the images associated with the three scalar components of Bethe sources. From top to bottom: 1) $g_{i}(\boldsymbol{s})$, for a magnetic coupling for TE incidence, and 2) $g_{c}(\boldsymbol{s})$, for a TM incidence; 3) $g_{s}(s)$, for an electric coupling for TE incidence. Real and imaginary parts of each image are normalized to their respective peak absolute value.

set to $1 \mathrm{~V} / \mathrm{m}$, the resulting image would correspond to a linear combination of electric and magnetic couplings

$$
\begin{aligned}
g_{\mathrm{TE}}(\boldsymbol{s}) & =\left[Y_{m}^{\tau}, Y_{m}^{\eta}\right] \boldsymbol{\alpha}_{2}^{m} g_{i}(\boldsymbol{s})+Y_{e} \alpha_{n}^{e} g_{s}(\boldsymbol{s}) \\
& =A_{\mathrm{TE}}^{m} g_{i}(\boldsymbol{s})+A_{\mathrm{TE}}^{e} g_{s}(\boldsymbol{s})
\end{aligned}
$$

where $\boldsymbol{\alpha}_{2}^{m}$ is the second column of $\boldsymbol{\alpha}^{m}$. The contribution from magnetic coupling can be extracted straightforwardly, by integrating $g_{\mathrm{TE}}(s)$ over a circular path $\gamma$ of radius $r$ centered at the peak of $\left|g_{\mathrm{TE}}(\boldsymbol{s})\right|$, which corresponds to the position of the aperture. The integration filters out the contribution given by the electric coupling, by virtue of the skew symmetry of $g_{s}(s)$, thus exposing the intensity of the TE magnetic contribution

$$
\hat{A}_{\mathrm{TE}}^{m}(\boldsymbol{s})=\frac{\oint_{\gamma} \mathrm{d} \boldsymbol{s} g_{\mathrm{TE}}(\boldsymbol{s})}{\pi^{2} \mathrm{~J}_{0}\left(k_{o} r\right)}=\left[Y_{m}^{\tau}, Y_{m}^{\eta}\right] \boldsymbol{\alpha}_{2}^{m} .
$$

In the same way, for TE electric coupling

$$
\hat{A}_{\mathrm{TE}}^{e}(\boldsymbol{s})=\frac{\oint_{\gamma} \mathrm{d} \boldsymbol{s} \sin \left(\vartheta_{s}\right) g_{\mathrm{TE}}(\boldsymbol{s})}{\mathrm{j} \pi^{2} \mathrm{~J}_{1}\left(k_{o} r\right)}=Y_{e} \alpha_{n}^{e} .
$$

A similar result holds for the TM case, with

$$
g_{\mathrm{TM}}(\boldsymbol{s})=\left[Y_{m}^{\tau}, Y_{m}^{\eta}\right] \boldsymbol{\alpha}_{1}^{m} g_{c}(\boldsymbol{s})=A_{\mathrm{TM}}^{m} g_{c}(\boldsymbol{s})
$$

providing a complementary information, for the first column $\boldsymbol{\alpha}_{1}^{m}$ of $\boldsymbol{\alpha}^{m}$, by means of

$$
\hat{A}_{\mathrm{TM}}^{m}(\boldsymbol{s})=\frac{\oint_{\gamma} \mathrm{d} \boldsymbol{s} g_{\mathrm{TM}}(\boldsymbol{s})}{4 \pi \mathrm{J}_{0}\left(k_{o} r\right)}=\left[Y_{m}^{\tau}, Y_{m}^{\eta}\right] \boldsymbol{\alpha}_{1}^{m} .
$$

These results imply that the proposed procedure does not result in the absolute polarizabilities of an aperture, but rather in linear combinations of their individual contributions, due to the unknown internal coupling functions. The distortion effect of the internal coupling could be overcome by having access to a larger number of receivers, in order to infer the aperture polarizabilities as common features shared by all the images computed from the received signals.

\section{CONCLusions}

We have shown that it is possible to infer the position of an aperture in a shielding, starting from signals received at its interior, when submitted to plane waves covering all possible directions of arrival. The procedure is reminiscent of inverse synthetic aperture radar imaging, and produces predictable spatial images centered on the aperture. The shape of the images can be related to the coupling mechanism, and ultimately on the dipole moments of Bethe sources. These results are expected to lead to the definition of a procedure for the identification of the type and orientation of apertures from automatic radiative tests.

\section{REFERENCES}

[1] K. Lee, EMP interaction: principles, techniques, and reference data: a handbook of technology from the EMP interaction notes. Taylor \& Francis/Hemisphere, 1986.

[2] M. Li, J. Drewniak, T. Hubing, R. DuBroff, and T. VanDoren, "Slot and aperture coupling for airflow aperture arrays in shielding enclosure designs," in Electromagnetic Compatibility, 1999 IEEE International Symposium on, vol. 1, 1999, pp. 35-39 vol.1.

[3] S.-H. Won and J.-G. Lee, "Shielding performance monitoring system using leaky coaxial cables," in Electromagnetics in Advanced Applications (ICEAA), 2013 International Conference on, Sept 2013, pp. 567-568.

[4] C. Baum, "Monitor for integrity of seams in a shield enclosure," Electromagnetic Compatibility, IEEE Transactions on, vol. 30, no. 3, pp. 276281, Aug 1988.

[5] H. Chen, H. Zhang, Z. Ni, and R. Li, "Research on imaging detection of RF leakage on the surface of spacecraft," in Electromagnetic Compatibility (EMC EUROPE), 2012 International Symposium on, Sept 2012, pp. $1-4$.

[6] D. L. Mensa, High Resolution Radar Imaging. Artech House, 1981.

[7] H. Bethe, "Theory of diffraction by small holes," Physical Review, vol. 66, no. 7-8, p. 163, 1944. 